\title{
COVID-19 y protocolo MAIZ como tratamiento profiláctico en Honduras, 2020: Artículo de revisión
}

\author{
COVID-19 and MAIZ protocol as prophylactic treatment in Honduras, 2020: Literature Review.
}

\begin{abstract}
Jhiamluka Zservando Solano Velázquez, $\odot^{1}$ César Tróchez Mejía, $\odot^{2}$ May Lin María Herrera Chang. $\bigodot^{3}$
'Médico Residente de Primer Año de Medicina Interna; Blackpool Victoria Hospital, North West Manchester, Maestrante de segundo año en Educación Médica; South Wales University, Reino Unido.

${ }^{2}$ Médico Especialista en Medicina Interna, Subespecialista en Gastroenterología y Alta-especialidad en Endoscopía Gastrointestinal; Hospital y Clínicas San Jorge; Hospital Medical Center; Hospital Tróchez Montalván; Tegucigalpa, Honduras.

${ }^{3}$ Doctora en Cirugía Dental, egresada de la Facultad de Odontología UNAH; Clínica Dental OdontoSalud; Comayagua, Honduras.
\end{abstract}

RESUMEN. Honduras se encuentra en el puesto 56 a nivel mundial con 4,151 muertes reportadas y 170,304 casos confirmados de COVID-19 hasta el 1 de marzo del 2021. Esta revisión bibliográfica se llevó a cabo con el propósito de brindar una perspectiva científica sobre el uso del protocolo MAIZ (Microdacyn, Azitromicina, Ivermectina y Zinc) propuesto para uso terapéutico y profiláctico durante la fase de infección precoz/respuesta viral y contactos asintomáticos de COVID-19. Se realizó una revisión de la literatura sobre los lineamientos de instituciones gubernamentales y organismos internacionales, artículos y estudios científicos en PubMed, The Lancet, SciELO. La búsqueda se limitó a los artículos en español e inglés publicados entre el año 2010 y el 1 de marzo del 2021 concluyendo que el protocolo MAIZ carece de evidencia científica que respalde su efectividad contra el COVID-19 y es necesario realizar ensayos clínicos para respaldar su uso de manera responsable.

Palabras clave. COVID-19, Honduras, Profilaxis.
Recibido: 02-08-2020 Aceptado: 24-03-2021 Primera vez publicado en línea: 22-04-2021 Dirigir correspondencia a: Dr. Jhiamluka Solano

Correo electrónico: jhiamv@gmail.com

Declaración de relaciones y actividades financieras y no financieras y conflictos de interés: ninguno.

DOl: https://doi.org/10.5377/rmh.v89i1.11577

๑ 2021 Autor(es): @ ( )

\section{INTRODUCCIÓN}

La familia de los Coronavirus produce enfermedades como el resfriado común, Síndrome Respiratorio Agudo Severo (SARS), Síndrome Respiratorio del Medio Oriente (MERS), entre otras. El Coronavirus 2 es una nueva cepa descubierta en el 2019 que produce el Síndrome Respiratorio Agudo Severo por Coronavirus 2 (SARS-CoV-2) recibiendo el nombre de Enfermedad por Coronavirus del 2019 (COVID-19) la cual ha desatado una pandemia sin precedentes. Para el 1 de marzo del 2021, la Organización Mundial de la Salud (OMS) confirmaba 113,820, 168 casos positivos, 2,527,891 muertes y 223 países afectados. ${ }^{1}$ Honduras junto al resto del continente americano, se ha vuelto el centro de la pandemia con $50,595,663$ casos confirmados hasta la fecha, por encima de Europa con 38,788,470 y Suroeste de Asia donde inició el brote con $13,539,176$ casos confirmados, ${ }^{2}$ siendo Brasil y Estados Unidos los países con las cifras más altas con 10,551,259 y $29,259,111$ de habitantes infectados respectivamente. Honduras se encuentra en la posición 69 del mundo ${ }^{3}$ con 4,151 muertes reportadas y 170,304 casos confirmados hasta el 1 de marzo del 2021. La búsqueda de tratamientos que previenen o curan la enfermedad se ha convertido en una carrera contra el reloj desde el inicio de la pandemia, siendo este un esfuerzo de toda la comunidad científica a nivel mundial para llevar a cabo investigaciones sobre el virus, ensayos clínicos sobre posibles tratamientos y vacunas, simulaciones y estrategias de prevención en salud pública, entre otras.

Las medidas de higiene y la cuarentena continúan estando vigentes desde hace más de 1,440 años como la principal medida de prevención para la propagación de una enfermedad infecciosa. ${ }^{4}$ Estas medidas deben ir de la mano de protocolos respaldados por la evidencia científica más actual, evitando tomar decisiones fundamentadas en información anecdótica olvidando el principio de la medicina basada en evidencia, descrito en 1996 por el Dr. David Sackett como "el uso consciente, explícito y juicioso de la mejor evidencia actual en la toma de decisiones sobre la atención de pacientes individuales", siendo 
esta individualización uno de los grandes retos que enfrentan los científicos frente al COVID19. ${ }^{5}$

Se llevó a cabo una revisión bibliográfica de documentación de instituciones gubernamentales y organismos internacionales, así como de artículos y estudios científicos con el propósito de brindar a la comunidad médica hondureña una perspectiva científica sobre el uso del protocolo Microdacyn, Azitromicina, Ivermectina y Zinc (MAIZ) de manera profiláctica o terapéutica en pacientes durante la fase de infección precoz/respuesta viral del COVID-19 y contactos asintomáticos. Se realizó búsqueda bibliográfica en PubMed, The Lancet, SciELO, páginas oficiales de organismos internacionales, gobiernos y del Estado de Honduras. Se utilizaron términos de búsqueda como COVID-19, Ivermectina, Azitromicina, Zinc, Microdacyn y Profilaxis. Se utilizó el método de bola de nieve para identificar recursos adicionales citados en los artículos consultados. La búsqueda se limitó a los artículos en español e inglés publicados entre el 2010 y el 1 de marzo del 2021. El 90\% de los estudios citados fueron publicados entre 2018-2020 y siendo la única excepción publicado en 2005 el estudio "super-oxidized water with neutral pH and disinfectant activity" por su relevancia científica en la discusión.

\section{PROTOCOLO MAIZ}

En Honduras, se ha propuesto por un grupo de médicos hondureños y adoptado por parte de las autoridades gubernamentales, ${ }^{6,7}$ el uso de un protocolo de tratamiento terapéutico durante la "Fase de Infección Precoz/Respuesta Viral del COVID-19" y de forma profiláctica para personas que son "contactos asintomáticos", el cual posteriormente fue denominado "MAIZ" por los medicamentos que contiene (Cuadro 1), ${ }^{8-10}$ junto a la hidroxicloroquina. Hasta la fecha la OMS y otros estudios publicados a nivel mundial aconsejan en contra del uso de antibióticos profilácticos en contactos asintomáticos y paciente con síntomas leves e indican su uso solamente en caso de sospecha de sobreinfección bacteriana. ${ }^{11,12}$ Este grupo de medicamentos forma parte del protocolo de atención del Gobierno de Honduras, ha sido distribuido para el manejo de contactos sintomáticos y pacientes positivos con síntomas leves con la hipótesis de prevención en la evolución del paciente a cuadros clínicos moderados o severos, disminución en el contagio y carga viral.

La Azitromicina es un antibiótico que pertenece a la familia de los macrólidos, utilizado mundialmente como tratamien- to empírico de primera línea en combinación con betalactámicos para manejo de pacientes con neumonía adquirida en la comunidad. ${ }^{12}$ Una de las complicaciones más comunes en pacientes con infección por Coronavirus es la sobreinfección bacteriana. El uso de Azitromicina se recomienda en presentaciones clínicas de neumonía moderada con signos y síntomas de hipoxemia y/o dificultad respiratoria con infiltrados pulmonares visibles en la rayos-x de tórax..$^{13}$ Aunque este antibiótico posee un efecto inmunomodulador que disminuye la reacción inflamatoria y antiviral ante el virus de la Influenza Tipo A H1N1 y ZIKA, no existen estudios que presenten niveles de evidencia científica suficientes para demostrar que este y otros antibióticos tengan un rol en el manejo del proceso viral sin sobreinfección bacteriana., ${ }^{4,14,15}$ Por lo tanto, su uso por si sola 0 en conjunto con hidroxicloroquina para tratamiento de COVID-19 en ausencia de sobreinfección bacteriana no está recomendado. ${ }^{16,17}$ Se reportaron efectos secundarios gastrointestinales, prolongación del segmento QT y la duración del potencial de acción monofásico, ${ }^{17}$ por ende, su uso ambulatorio debe ser evitado al no tener monitoreo constante con un electrocardiógrafo. Se demostró que los pacientes que recibían un curso de cinco días tenían mayor riesgo de muerte súbita cardíaca comparado a los que recibieron amoxicilina o ningún antibiótico. Los estudios que presentaban algún efecto positivo después de un abordaje de Graduación de Evaluación, Desarrollo y Recomendaciones (GRADE) se clasificaron como nivel de evidencia baja debido a los tamaños de la muestra, co-intervenciones y riesgo de sesgo debido a limitadas metodologías. ${ }^{18,19}$

No existen hasta la fecha ensayos clínicos controlados que evalúen el uso de antibióticos de manera empírica en pacientes con SARS-CoV-2 u otros coronavirus, mucho menos de manera profiláctica. El Colegio Americano de Cardiología, Agencia Europea de Medicamentos (EMA) y la OMS advierten sobre los riesgos presentes en este tratamiento debido a la falta de evidencia que respalde sus beneficios. ${ }^{17,20}$ Sin embargo, esto no es tarea fácil, por una parte, es necesaria la evidencia científica que confirme que el beneficio de este tratamiento ha superado los riesgos y por otra, los ensayos clínicos necesarios para esto pueden ser difíciles de planificar, implementar y en última instancia generar evidencia científica sólida en poco tiempo bajo presión durante la pandemia. ${ }^{17}$

Cuadro 1. Medicamentos del protocolo MAIZ sobre el manejo de manera profiláctica para evitar complicaciones en pacientes con COVID-19 en Honduras (Adaptado de referencias 8-10).

\begin{tabular}{ccc}
\hline & & \multicolumn{2}{c}{ Protocolo MAIZ } \\
Medicamento & Tipo & Dosis \\
\hline Microdacyn & Solución esterilizante y antiséptica & $10 \mathrm{ml}$ cada 6 horas en forma de gárgaras durante 10 días \\
Azitromicina & Antibiótico & $500 \mathrm{mg}$ Vía oral una vez al día por 5 días \\
Ivermectina & Antiparasitario & $6 \mathrm{mg}$ Vía oral una vez al día por 5 días \\
Zinc & Mineral & $50 \mathrm{mg}$ Vía oral una vez al día por 10 días \\
\hline
\end{tabular}


El Microdacyn es una solución esterilizante y antiséptica que reduce la carga viral de adenovirus y $\mathrm{VIH}$ en superficies no corporales. ${ }^{21}$ Está compuesto por agua en un $99.99 \%$ e iones adicionales. ${ }^{22}$ Su mecanismo de acción se basa en desnaturalización de proteínas y fragmentación de carbohidratos y lípidos, además de la alteración viral de las cápsides, DNAsas y RNasas. ${ }^{23}$ Debido a la transmisión directa de SARS-CoV-2 por contacto con saliva ${ }^{24}$ se han realizado análisis a pacientes SARS-CoV-2 positivo, cuyos resultados demuestran una amplia expresión del receptor de Enzima Convertidora de Angiotensina 2 (ECA2) en saliva. Su nivel en diferentes células epiteliales de la mucosa oral, especialmente lengua y glándulas salivales menores es mayor que en intestinos, corazón e incluso pulmones, la presencia de SARS-CoV-2 RNA se encuentra en saliva incluso antes de que lesiones pulmonares aparezcan. ${ }^{25,26}$ El enjuague oral preoperatorio con clorhexidina, peróxido de hidrógeno, yodo povidone y cloruro de cetilpiridinio, entre otros, demuestra eficaz reducción de microorganismos en cavidad oral, ${ }^{24,26,27} \mathrm{sin}$ embargo, no existe evidencia directa del impacto que puedan ejercer sobre la carga viral de SARS-CoV-2, y a su vez se advierte la posible irritación y ulceración en mucosa oral y nasal.

En el Microdacyn, compuestos como el hipoclorito de sodio, aparte de su efectividad contra bacterias, virus, hongos y esporas, tiene efectos tóxicos en tejidos vitales ${ }^{23}$ y puede causar irritación en mucosa, broncoespasmo, neumonía, y edema pulmonar. ${ }^{28} \mathrm{El}$ dióxido de cloro $\left(\mathrm{ClO}_{2}\right)$ ha sido usado en enjuagues bucales e irrigadores de conductos radiculares, y se reporta que incluso en bajas concentraciones puede inducir a la detención del ciclo celular en fibroblastos gingivales en seres humanos. ${ }^{29}$ Por otra parte, el ácido hipocloroso ha sido recomendado, entre otros, por la Agencia de Protección Ambiental de Estados Unidos (USEPA, por sus siglas en inglés) como desinfectante contra SARS-CoV-2. Es un compuesto no tóxico al contacto, bien tolerado por tejidos orales y con efectos antiinflamatorios. ${ }^{29,30}$ No obstante, estas recomendaciones deben validarse con ensayos clínicos que evalúen su eficacia y seguridad previo al uso como parte de esquemas terapéuticos preventivos.

En cuanto a la Ivermectina, este es un antiparasitario de amplio espectro aprobado por la FDA que ha demostrado inhibir la replicación de virus in-vitro como ser el virus del dengue y HIV1. En relación con el SARS-CoV esta puede disociar al heterodímero IMPa/ $\beta 1$ preformado, responsable del transporte nuclear de las cargas de proteínas virales por lo que sugieren que la actividad inhibidora del transporte nuclear puede ser efectiva contra el SARS-CoV-2 demostrado igualmente in-vitro. ${ }^{31}$ Como se señaló, la Ivermectina fue el foco de un reciente ensayo clínico de fase III en pacientes con dengue en Tailandia, en el que se encontró que una sola dosis no producía beneficio clínico alguno. ${ }^{32}$ Múltiples medicamentos son potentes inhibidores de citocromo P 450 3A4, la principal vía metabólica de la Ivermectina, el uso concomitante de estos medicamentos puede resultar en el incremento sistémico de la Ivermectina y provocar eventos adversos como neurotoxicidad especialmente debido a las altas dosis recomendadas para poder lograr el efecto antiviral e antiinflamatorio deseado expuesto en varios estudios. ${ }^{32,33}$ Es ideal la consideración de ensayos clínicos bien realizados informados por modelos farmacocinéticos para validar el impacto antes de implementar el uso de ivermectina para tratar el SARS-CoV-2.34-37

El Zinc (Zn) ha demostrado disminuir la replicación de otros coronavirus, pero no existe evidencia científica sobre un efecto directo anti-SARS-CoV-2. ${ }^{38}$ Los datos obtenidos demuestran que los niveles adecuados de zinc aumentan la reactividad inmune. Aunque los efectos terapéuticos del Zn se consideran inconsistentes, los datos existentes basados en evidencia indican la eficiencia de la suplementación de $\mathrm{Zn}$ y la mejora de niveles de $\mathrm{Zn}$ en la prevención de la neumonía y sus complicaciones debido al efecto antiinflamatorio del mismo.

Existen ciertas indicaciones indirectas e hipótesis ${ }^{39}$ del posible efecto antiviral de Zn contra SARS-CoV-2, aunque su relevancia biomédica aún no se ha estudiado. En vista de los datos recientes sobre el curso clínico de la enfermedad, se cree que los niveles adecuados de $\mathrm{Zn}$ pueden tener un efecto protector como terapia adyuvante de COVID-19 a través de la reducción de la inflamación pulmonar, mejora del aclaramiento mucociliar, prevención de la lesión pulmonar inducida por el ventilador, modulación de inmunidad antibacteriana y antiviral especialmente en ancianos. ${ }^{40}$ Se requieren estudios clínicos y experimentales adicionales para dilucidar el papel potencial de la deficiencia de Zn en la susceptibilidad a COVID-19, así como los efectos de la suplementación con $\mathrm{Zn}$ y los mecanismos subyacentes.

\section{CONCLUSIÓN}

El protocolo MAIZ actualmente utilizado en Honduras carece de estudios que respalden su implementación y va en contra de las directrices establecidas por la Organización Mundial de la Salud. Dentro del "Manejo Inteligente" de la pandemia establecido en Honduras, se realizó la compra y distribución de los medicamentos que conforman el protocolo MAIZ con el objetivo de "homogeneizar" el tratamiento y distribuir en los distintos niveles de atención, evitando así principalmente que los enfermos lleguen a los hospitales. Es importante resaltar que este protocolo no ha sido publicado en ninguna revista médica nacional o internacional donde se pueda evaluar la metodología utilizada en algún ensayo clínico aleatorio y sus resultados e interpretación estadística para replicar y respaldar la hipótesis sobre la posible eficacia de estos protocolos. A pesar de esto, las autoridades gubernamentales aseguran que el uso del protocolo MAIZ provoca una reducción de carga viral en más del $90 \%$ sin haber realizado ningún estudio previo que sustente esta información. Como resultado, este tipo de propuestas carente de suficiente evidencia científica provoca que la población deje a un lado el concepto básico de prevención, haciendo uso de mascarillas y medidas de bioseguridad, así como el distanciamiento físico que ha demostrado impacto en la disminución del número de contagios. En ningún otro país se utiliza este protocolo de forma indiscriminada y como manejo estandarizado profiláctico o terapéutico, sin mencionar que hasta la fecha no se ha comprobado que algún medicamento 
disponible en el mercado prevenga la infección por COVID-19. Es importante reconocer que existen diversos estudios ${ }^{17,41}$ que han evaluado el uso de los medicamentos propuestos en el protocolo MAIZ de manera individual sin encontrar beneficios para su uso, así mismo, existen estudios enfocados en la posible efectividad de otros medicamentos.

Es importante mantener como eje central del manejo terapéutico a las guías proporcionadas por la Organización Mundial de la Salud que se encuentran en constante actualización a medida emerge nueva evidencia científica. Cada médico de primera línea deberá hacer uso de medicina basada en evidencia para el manejo de pacientes de forma individualizada con el fin de disminuir la mortalidad. Por otro lado, el gobierno de
Honduras debe promover la orientación adecuada por parte de expertos que se apoyen en la evidencia científica más actual y que carezcan de conflictos de interés al momento de la toma de decisiones para garantizar el derecho universal a la salud. Es importante la reflexión sobre la pandemia y preguntarse, ¿Qué hace a un médico o científico experto en SARS-CoV-2? La comunidad científica mundial todavía no tiene la respuesta.

\section{DETALLE DE AUTORES}

1.Jhiamluka Zservando Solano V. jhiamv@gmail.com ${ }^{2}$ César Tróchez Mejía

${ }^{3}$ May Lin María Herrera Chang

\author{
trochez48@hotmail.com \\ mayherrera.ch@gmail.com
}

\section{REFERENCIAS}

1. World Health Organization. Coronavirus disease (COVID-19) pandemic [Internet]. Ginebra: WHO; 2020 [consultado 1 marzo 2021]. Disponible en: https://www.who.int/emergencies/diseases/novel-coronavirus-2019

2. World Health Organization. WHO Coronavirus (COVID-19) Dashboard [Internet]. Ginebra: WHO; 2020 [consultado 1 marzo 2021]. Disponible en: https://covid19.who.int/

3. WorldoMeter. COVID-19 coronavirus pandemic 2020 [Internet]. Delaware: Worldometer; 2020 [consultado 14 noviembre 2020]. Disponible en: https://www.worldometers.info/coronavirus/

4. Abd El-Aziz TM, Stockand JD. Recent progress and challenges in drug development against COVID-19 coronavirus (SARS-CoV-2) - an update on the status. Infect Genet Evol [Internet]. 2020 [consultado 20 junio 2020]; 83:104327. Disponible en: https://www.ncbi.nlm.nih.gov/pmc/ articles/PMC7166307/

5. Isaacs D. Evidence $\square$ based medicine. J Paediatr Child Health. 2014;50(8): $579-80$

6. Plataforma Todos Contra el COVID-19 (HN). Consideraciones epidemiológicas sobre la tasa semanal de casos reportados por millón de habitantes de pruebas PCR-TR realizadas por el Laboratorio de Vigilancia de la Salud, Secretaría de Salud de Honduras [Internet]. Tegucigalpa: PTCC; 2020 [consultado 3 junio 2020]. Disponible en: http:// www.desastres.hn/COVID-19/Plataforma/13_Plataforma\%20Todos $\% 20$ Contra\%20el\%20COVID Informe 06.07.2020.pdf

7. Secretaría de Salud $(\mathrm{HN})$. Protocolo de manejo clínico del paciente adulto con covid-19 según las etapas de la enfermedad en las redes de servicios de salud [Internet]. Tegucigalpa: Secretaría de Salud; 2020 [consultado 20 junio 2020]. Disponible en: https://n9.cl/4kobv

8. Plataforma Todos Contra el COVID-19 (HN). Guía médica de diagnóstico clínico laboratorial y manejo del Covid-19 [Internet]. Tegucigalpa: PTCC; 2020 [consultado 2 julio 2020]. Disponible en: http://www.desastres.hn/ COVID-19/Plataforma/Guia_DiagnosticoManejo_COVID19_27042020. pdf

9. Inversión Estratégica de Honduras. Entregados medicamentos para el protocolo MAIZ [Internet]. Tegucigalpa: INVESTH; 2020. [consultado 2 julio 2020]. Disponible en: http://www.investhonduras.hn/2020/06/20/ entregados-medicamentos-para-el-protocolo-maiz/

10. Sistema Nacional de Gestión de Riesgo (HN). MAíZ PACK: tratamiento de 10 días [Internet]. Tegucigalpa: SINAGER; 2020. [consultado 2 julio 2020]. Disponible en: https://www.megalabs.global/wp-content/ uploads/2020/06/Animacion-MAIZ-Pack-Gobierno-de-Honduras.pdf

11. World Health Organization. Clinical Management of Covid-19 [Internet]. Ginebra: Suiza; 2020. [consultado 20 junio 2020]. Disponible en: https:// apps.who.int/iris/bitstream/handle/10665/332196/WHO-2019-nCoVclinical-2020.5-eng.pdf?sequence $=1$ \&isAllowed

12. Razai MS, Doerholt $K$, Ladhani $S$, Oakeshott P. Coronavirus disease 2019 (COVID-19): a guide for UK GPs. BMJ [Internet]. 2020 [consultado 2 julio 2020];368:m800. Disponible en: https://www.bmj.com/content/ bmj/368/bmj.m800.full.pdf

13. González-Martín L, Librán Peña A, Andrés de Álvaro M. Antibioterapia empírica en la neumonía adquirida en la comunidad: ¿es hora de prescindir de los macrólidos? Acta Pediatr Esp. 2020;78(1-2):e1-e5

14. Infectología y COVID-19: [guías] clínicas provisionales para la detección y manejo del paciente con COVID-19 en Honduras [Internet]. Tegucigalpa; 2020 [consultado 2 julio 2020]. Disponible en: http://www.desastres. hn/COVID-19/covid19guiasprovisionalesresumidasyextendidas/ Infectologia COVID19 VersionExtendida 27032020.pdf

15. Gbinigie K, Frie K. Should azithromycin be used to treat COVID-19? A rapid review. BJGP Open. 2020;4(2): bjgpopen20X101094.

16. Sultana J, Cutroneo PM, Crisafulli S, Puglisi G, Caramori G, Trifiró G. Azithromycin in COVID-19 patients: pharmacological mechanism, clinical evidence and prescribing guidelines. Drug Saf. 2020;43(8):691-8.

17. Horby PW, Roddick A, Spata E, Staplin N, Emberson JR, PessoaAmorim G, et al. Azithromycin in hospitalised patients with COVID-19 (RECOVERY): a randomised, controlled, open-label, platform trial. MedRxiv [Preprint]. 2020.[Posteado 14 diciembre 2020; consultado 21 enero 2021] Disponible en: https://www.medrxiv.org/content/10.1101/20 20.12.10.20245944v1

18. American Society of Health System Pharmacists. Assessment of evidence for COVID-19-related treatments [Internet]. Bethesda: ASHP; 2020 [consultado 2 julio 2020]. Disponible en: https://www.fip.org/files/ content/priority-areas/coronavirus/mo-resources/ASHP-COVID-19Evidence-Table-03-21-20.pdf

19. Bhimraj A, Morgan RL, Shumaker AH, Lavergne V, Baden L, Cheng VC, et al. Infectious Diseases Society of America guidelines on the treatment and management of patients with COVID-19. Clin Infect Dis [Internet]. 2020 [consultado 2 julio 2020];27:ciaa478. Disponible en: https://www. idsociety.org/practice-guideline/covid-19-guideline-treatment-andmanagement/

20. Pani A, Lauriola M, Romandini A, Scaglione F. Macrolides and viral infections: focus on azithromycin in COVID-19 pathology. Int J Antimicrob Agents. 2020; 56(2):106053.

21. Alhazzani W, Møller MH, Arabi YM, Loeb M, Gong MN, Fan E, et al. Surviving sepsis campaign: guidelines on the management of critically ill adults with coronavirus disease 2019 (COVID-19). Intensive Care Med. 2020; 28:1-34

22. Landa-Solis C, González-Espinosa D, Guzmán-Soriano B, Snyder M, Reyes-Terán G, Torres K, et al. Microcyn: a novel super-oxidized water with neutral pH and disinfectant activity. J Hosp Infect. 2005;61(4):291-9.

23. Mena-Mendivil ED, Flores-Treviño JJ, Rodríguez-Delgado I, De la Garza Ramos M, Torre-Martínez HH, Martínez-González GI. Antimicrobial effect of microdacyn 60 , OxOral, and sodium hypochlorite $5.25 \%$ in anaerobic bacteria. J Pharmacognosy Phytother. 2013;5(10):178-82

24. Barca I, Cordaro R, Kallaverja E, Ferragina F, Cristofario MG. Management in oral and maxillofacial surgery during the COVID-19 pandemic: our experience. Br J Oral Maxillofac Surg. 2020;58(6):687-91.

25. Baghizadeh Fini M. Oral saliva and COVID-19. Oral Oncol. 2020; 108:104821

26. Herrera $D$, Serrano J, Roldán $S$, Sanz $M$. Is the oral cavity relevant in SARS-CoV-2 pandemic? Clin Oral Investig. 2020; 23:1-6.

27. Baghizadeh Fini M. What dentists need to know about COVID-19. Oral 
Oncol. 2020; 105:104741.

28. Aronson J.K. Meyler's side effects of drugs. The International Encyclopedia of Adverse Drug Reactions and Interactions. 16th ed. Ámsterdam: Elsevier; 2016.

29. Lafaurie GI, Zaror C, Díaz-Báez D, Castillo DM, De Ávila J, Trujillo TG, et al. Evaluation of substantivity of hypochlorous acid as an antiplaque agent: a randomized controlled trial. Int J Dent Hyg. 2018;16(4):527-534.

30. Block MS, Rowan BG, Hypochlorous acid: a review. J Oral Maxillofac Surg. 2020;78(9):1461-66.

31. Caly L, Druce JD, Catton MG, Jans DA, Wagstaff KM. The FDA-approved drug ivermectin inhibits the replication of SARS-CoV-2 in vitro. Antiviral Res. 2020; 178:104787.

32. Caly L, Druce JD, Catton MG, Jans DA, Wagstaff KM. The FDAapproved drug ivermectin inhibits the replication of SARS-CoV-2 in vitro. Antiviral Res. [Internet]. 2020 [Consultado 2 de julio 2020]; 178:104787. Disponible en: https://pubmed.ncbi.nlm.nih.gov/32251768/

33. Vallejos J, Zoni R, Bangher M, Villamandos S, Bobadilla A, Plano F, et al. Ivermectin to prevent hospitalizations in patients with Covid-19 (IVERCOR-COVID19): a structured summary of a study protocol for a randomized controlled trial. Trials. 2020;21(1):965.

34. Gupta D, Sahoo AK, Singh A. Ivermectin: potential candidate for the treatment of COVID 19. Braz J Infect Dis [Internet]. 2020 [consultado 9 noviembre 2020];24(4):369-371. Disponible en: https://www. sciencedirect.com/science/article/pii/S1413867020300817?via\%3Dihub

35. Chaccour C, Hammann F, Ramón-García S, Rabinovich NR. Ivermectin and Covid-19: keeping rigor in times of urgency. Am J Trop Med Hyg. 2020;102(6):1156-1157.

36. Organización Panamericana de la Salud. Recomendación sobre uso ivermectina para el tratamiento de COVID-19 [Internet]. Washington D.C.: OPS; 2020 [consultado 23 junio 2020]. Disponible en: https://docs. bvsalud.org/biblioref/2020/06/1100598/ops-tratamiento-ivermectinajunio-22-2020.pdf

37. Heidary $F$, Gharebaghi R. Ivermectin: a systematic review from antiviral effects to COVID-19 complementary regimen. J Antibiot. 2020;73(9):593602.

38. Skalny AV, Rink L, Ajsuvakova OP, Aschner M, Gritsenko VA, Alekseenko SI, et al. Zinc and respiratory tract infections: perspectives for COVID-19 (Review). Int J Mol Med [Internet]. 2020 [Consultado 1 febrero 2021];46(1):17-26. Disponible en: https://www.ncbi.nlm.nih.gov/pmcl articles/PMC7255455/

39. Mayor-Ibarguren A, Busca-Arenzana C, Robles-Marhuenda Á. A
Hypothesis for the possible role of zinc in the immunological pathways related to COVID-19 infection. Front Immunol [Internet]. 2020 [consultado 10 julio 2020]; 11:1736. Disponible en: https://doi.org/10.3389/ fimmu.2020.01736

40. Wessels I, Rolles B, Rink L. The potential impact of zinc supplementation on COVID-19 pathogenesis. Front Immunol [Internet]. 2020 [Consultado 10 julio 2020]; 11:1772. Disponible en: https://www.ncbi.nlm.nih.gov/pmc/ articles/PMC7365891/

41. National Institutes of Health. The COVID-19 Treatment Guidelines Panel's Statement on the Use of Ivermectin for the Treatment of COVID-19. [Internet]. 2020 [consultado 13 noviembre 2020]. Disponible en: https:// files.covid19treatmentguidelines.nih.gov/guidelines/section/section_106. pdf

ABSTRACT. Honduras is in the position 68th worldwide with 4 151 reported deaths and 170, 304 confirmed cases of COVID-19 as of March $1^{\text {st }}, 2021$. This literature review was conducted to provide a scientific perspective on the use of the MAIZ protocol (Microdacyn, Azithromycin, Ivermectin and Zinc) proposed for therapeutic and prophylactic uses during the early infection/viral response phase and asymptomatic contacts of COVID-19. A literature review was conducted from government institutions and international agencies guidelines, scientific articles, and studies in PubMed, The Lancet, SciELO. The review was limited to articles in Spanish and English published between 2010 and the $1^{\text {st }}$ of March 2021, concluding that the MAIZ protocol lacks scientific evidence that supports the effectiveness against COVID-19, and clinical trials are needed to support its use responsibly.

Keywords. COVID-19, Honduras, Prophylaxis. 